\title{
Relative Contributions of History-taking, Physical Examination, and Laboratory Investigation to Diagnosis and Management of Medical Outpatients
}

\author{
J. R. HAMPTON, M. J. G. HARRISON, J. R. A. MITCHELL, J. S. PRICHARD, CAROL SEYMOUR
}

British Medical fournal, 1975, 2, 486-489

\section{Summary}

To evaluate the relative importance of the medical history, the physical examination, and laboratory investigations in the diagnosis and management of medical outpatients some physicians recorded their diagnosis and a prediction of the method of management after reading the patient's referral letter, again after taking the history, and again after performing the physical examination. These diagnoses and predictions were compared with the diagnosis and method of management which had been adopted two months after the patient's initial attendance. A diagnosis that agreed with the one finally accepted was made after reading the referral letter and taking the history in 66 out of 80 new patients; the physical examination was useful in or.ay seven patients, and the laboratory investigations in a further seven.

In only one of six patients in whom the physician was unable to make any diagnosis after taking the history and examining the patient did laboratory investigations lead to a positive diagnosis.

\section{Introduction}

The making of a medical diagnosis depends on three things: the history obtained from the patient, the signs noticed on physical examination, and the results of laboratory investigations. Platt (1947) claimed that a diagnosis could be achieved by history-taking alone in the majority of patients, but books on clinical methods for medical students still devote much more space to eliciting physical signs than to history taking, and the colossal increase in the work load of all service departments shows vividly how dependent we have become on laboratory investigations. We report here an attempt to measure the relative importance of the patient's history, the physical examination, and the laboratory investigations in arriving at a

Department of Medicine, Nottingham University

J. R. HAMPTON, D.M., M.R.C.P., Consultant Physician and Senior Lecturer in Medicine

J. R. A. MITCHELL, M.D., F.R.C.P., Professor of Medicine

Department of Neurology, Middlesex Hospital

M. J. G. HARRISON, D.M., M.R.C.P., Consultant Neurologist

Nuffield Department of Medicine, Radcliffe Infirmary, Oxford

J. S. PRICHARD, B.M., M.R.C.P., Senior Registrar

Royal Postgraduate Medical School, London

CAROL SEYMOUR, B.M., M.R.C.P., Senior Registrar diagnosis in a medical outpatient clinic. We asked physicians to record their diagnosis after taking the patient's history and to record it again after making a physical examination. By comparing these diagnoses with the ultimate one reached after laboratory investigations we have been able to show just how important the history is.

It is, of course, obvious that a final diagnosis cannot always be made with certainty even after detailed laboratory studies. Of more importance is what happens to the patient, and we have therefore also attempted to find out to what extent the management of the patient, as opposed to the making of a diagnosis, depends on the history, the examination, and the laboratory investigations.

\section{Methods}

During a four-month period each physician working in a weekly general medical clinic completed a standard form for each new patient he saw. The following questions were answered at set stages of the interview and examination.

\section{GENERAL PRACTITIONER'S DIAGNOSIS}

Having read the referral letter from the general practitioner the physician wrote down the practitioner's diagnosis. If no diagnosis was offered, then the patient's complaint was entered instead.

\section{DIAGNOSIS AFTER TAKING THE HISTORY}

After taking a complete history from the patient the physician wrote down his own diagnosis. A choice of three possibilities was allowed, with a fourth heading for "don't know." "Confidence scores" were attached to each possible diagnosis, with the points making a total of 10 . Thus a typical answer to this question was:

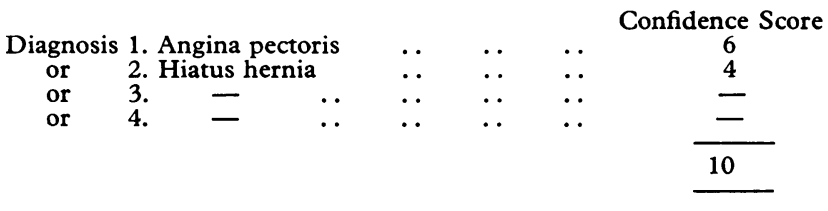

If the physician could make no diagnosis after taking the history then he allocated a confidence score of 10 to "don't know." 


\section{PREDICTED MANAGEMENT AFTER TAKING THE HISTORY}

Before examining the patient, the physician indicated which of the following possible courses of action he thought would be taken:

(a) No action required: the patient would be returned to the care of his general practitioner.

(b) The patient would need to be followed up in the medical outpatient clinic without treatment, awaiting further progress of his condition.

(c) The patient would need treatment under supervision in the outpatient clinic.

(d) The patient would need admission to hospital, either urgently or from the waiting list.

(e) The patient would need to be referred to another specialist clinic.

\section{DIAGNOSIS AFTER THE EXAMINATION}

Having examined the patient the physician again recorded his diagnosis. As before, he was allowed three possible diagnoses and a "don't know" category, and was asked to allocate confidence scores to his diagnosis.

\section{PREDICTED MANAGEMENT AFTER THE EXAMINATION}

The physician again indicated which of the five possible methods of management listed under $3 a-e$ he now believed would be followed.

\section{INVESTIGATIONS ORDERED}

The investigations requested were listed under three headings: essential, desirable, and routine. No advice was given in the use of these categories, and each physician made his own decision on whether any particular investigation was essential, whether it was desirable, or whether it was merely routine.

\section{Analysis of Records}

Two months after the patient had first attended the clinic the case records were analysed and the following points recorded:

(1) The final diagnosis of a specific pathological condition reached at that time was noted, and this was classified as "confident" or "probable." In some cases no final conclusion had been reached, and for these the category "no satisfactory diagnosis" was used.

Comparison with the form which had been completed when the patient first attended the clinic showed which of the following possibilities had occurred: (a) The final diagnosis was the same as that made by the general practitioner. (b) The final diagnosis differed from that made by the general practitioner but was the same as the diagnosis made by the physician after taking the history. (c) The final diagnosis differed from that made by the physician after taking the history, but was the same as that which he made after the physical examination. (d) The final diagnosis differed from that made after the physical examination and was made only as a result of laboratory investigation.

(2) The management of the patient at this time was classified as one of the following: (a) The patient had been returned to the care of his general practitioner. (b) The patient was being followed up in the outpatient clinic without treatment. (c) The patient was being treated and supervised in the outpatient clinic. (d) The patient had been admitted to hospital, either urgently or from the waiting list. (e) The patient had been referred to another outpatient clinic.

By comparison with the form completed during the patient's first visit to the clinic it was thus clear whether the management had been as predicted by the physician after taking the history. If on the other hand this prediction had been wrong, it could be seen whether the prediction made after the physical examination had been correct. If not, the final management of the patient must have depended entirely on the results of the investigations.

(3) The results of the investigations which had been ordered were checked, and the numbers of normal and abnormal results which had been obtained in the three categories (essential, desirable, and routine) were recorded. For the purposes of recording the numbers of investigations ordered, tests which could be done on a single sample by one process (for example, automated blood urea and electrolyte estimations) were recorded as one investigation. At the time of the study haematological investigations were not carried out in this way, and a haemoglobin and a white cell count were therefore recorded as separate investigations.

\section{Results}

\section{THE PATIENTS STUDIED}

Questionnaires were completed for the 123 new patients who attended the clinic during this four-month period. Three of these were inadequately completed and were therefore discarded.

Of the remaining 120 patients 40 had been referred by their general practitioners with a diagnosis of high blood pressure. This condition was known to be a special interest of the unit, and though a special clinic was held for hypertensive patients some practitioners referred their patients to the general clinic. Forms on these patients were completed, but as a diagnosis had already been made on the basis of a physical sign elicited by the referring practitioner before the patient had attended the clinic they have been excluded from the analysis described here. The residual group of 80 patients form the basis of this study and the problems with which they presented covered the whole range of conditions seen in a general medical clinic.

\section{ANALYSIS OF THE QUESTIONNAIRE}

\section{Making the Diagnosis}

Table I shows the number of patients in whom the diagnosis was changed during the sequence of history-taking, physical examination, and laboratory investigation. The total exceeds the number of patients in the study as in some cases the diagnosis was changed more than once.

TABLE I-Effect of History, Physical Examination, and Laboratory Investigations on the Diagnosis

Referring practitioner's diagnosis unchanged Diagnosis changed after history taking Diagnosis changed after physical examination Diagnosis changed after laboratory investigation

$$
\begin{array}{llr} 
& \multicolumn{2}{c}{\text { Number of patients }} \\
. & \ldots & 37 \\
\ldots & \ldots & 34 \\
. . & \ldots & 6 \\
& & 7
\end{array}
$$

A wide variety of conditions is included in the group of 37 patients in whom the final diagnosis made by the clinic physician was the same as that made by the referring practitioner. The group includes four patients sent up because the practitioner could not make a firm diagnosis and in whom no final diagnosis had been made two months later.

On the basis of the history the clinic physician reached a diagnosis which differed from that of the general practitioner in 34 patients. Of these 34 patients 11 had been referred by their practitioners because of specific symptoms such as breathlessness, chest or abdominal pain, palpitations, and so on, and no diagnosis had been offered by the referring practitioner. Some patients had been referred for opinions about physical signs (one with a lump in the neck, and another with coating of the tongue), and some came without adequate referral letters. 
In all these instances no referral diagnosis was offered. In 13 patients the practitioner had made a diagnosis which the physician considered incorrect after taking his own history.

In only six patients out of the 80 was the diagnosis made by the physician after he had taken the patient's history changed as a result of the examination. All six patients had cardiovascular problems: in two, heart murmurs had been heard by the practitioner, bi t the history gave no clue to their nature; two had been diagn osed as having ischaemic heart disease on the basis of their histories, but after examination they were considered respectively to have hypertensive heart disease and cardiomyopathy; one patient who had been thought to have hypertensive heart failure was found to have aortic stenosis; and one patient thought to have deep venous thrombosis in the leg was found to have superficial phlebitis and osteoarthritis of the knee.

Of the seven patients in whom laboratory investigations had been required to achieve the final diagnosis two had myeloma, one had sarcoidosis, and one had severe osteoarthritis of the spine. Two patients in whom a firm diagnosis had not been achieved at the end of the examination were found on investigation to have no organic disease, and one patient, thought as a result of history and examination to have a peptic ulcer, was found to have a rormal barium meal examination and a firm diagnosis of depression was later made. Thus the laboratory investigations had provided positive results which had been essential for the diagnosis in only four out of the 80 patients.

\section{Effect of the Physical Examination on the Diagnostic Confidencc Score}

In 55 patients the physical examination did nothing to change the physician's confidence in his diagnosis. This group included patients in whom the physician was completely confident of his diagnosis (for example, in one patient a score of 10 was given after taking the history to basilar artery insufficiency); patients in whom the physician was partially confident of his diagnosis (for example, diagnosis scores of anxiety, 7, and thyrotoxicosis, 3 , and patients in whom the physician was completely nonconfident of the diagnosis (scores of 10 given to "don't know").

In 19 patients the examination increased the physician's diagnostic confidence (for example, gout, 7, "don't know," 3, was changed to gout, 10 , while prolapsed intervertebral disc, 6 , "don't know," 4, was changed to prolapsed disc, 9, "don't know," 1). In six patients the examination reduced the physician's confidence (for example, myocardial infarction, 10, was changed to myocardial infarction, 7, pulmonary embolism, 2, “don't know," 1).

\section{Results of Laboratory Investigations}

Table II shows the numbers of laboratory investigations that were requested. Of 160 that were considered essential $40 \%$ proved abnormal; of 116 considered desirable $26 \%$ were abnormal, and of 202 considered routine $10 \%$ were abnormal.

Individual physicians showed very different attitudes to the use of investigations. The average number of investigations per patient was 6 , but the range among different physicians was 4.6-10.0 per patient. The physician who requested an average of 10.0 investigations per patient considered $59 \%$ of his requests as essential. Most of the physicians considered about one-third of their investigations to be essential, but one physician only included $14 \%$ of his investigations in this category. In the routine group most requests were for tests such as haemoglobin, erythrocyte sedimentation rate, or chest $x$-ray, but some special investigations were included in this category by some physicians. For example, in one case a peptic ulcer had been diagnosed on the basis of the history with a confidence score of 10 . A barium meal examination (which demonstrated an ulcer) had been performed as a routine test, since the physician considered that it would not affect the management of the patient.

Overall, $24 \%$ of the investigations yielded abnormal results. Among the different physicians the highest yield of abnormal results was $34 \%$ of the total requested, and the lowest was $11 \%$.

TABLE II-Laboratory Investigations Ordered in 80 Patients Studied

\begin{tabular}{|c|c|c|c|c|c|}
\hline & & & \multicolumn{3}{|c|}{ Result } \\
\hline & & & Normal & Abnormal & Total \\
\hline \multirow[t]{2}{*}{$\begin{array}{l}\text { Essential } \\
\text { Desirable } \\
\text { Routine }\end{array}$} & $\begin{array}{l}. \\
\cdots \\
.\end{array}$ & $\begin{array}{l}\cdots \\
\cdots\end{array}$ & $\begin{array}{r}96 \\
86 \\
181\end{array}$ & $\begin{array}{l}64 \\
30 \\
21\end{array}$ & $\begin{array}{l}160 \\
116 \\
202\end{array}$ \\
\hline & Total & . & 363 & 115 & 478 \\
\hline
\end{tabular}

The physician's confidence in his diagnosis was not reflected in his use of laboratory investigations. In the 41 patients in whom the physician made a confident diagnosis (score 10 or 9) an average of 5.5 investigations was ordered, and $21 \%$ of these investigations gave abnormal results. In the 23 cases in which the physician was not confident (confidence score for main diagnosis 6 or less, or "don't know" score of 10) an average of 5.5 investigations was again ordered, and $31 \%$ were found to be abnormal.

Furthermore, the number of investigations which had been performed when the patient attended the clinic was not directly related to the confidence score which could be attached to the final diagnosis made when the patient's notes were reviewed two months later. In those patients in whom the two-month diagnosis was recorded as "confident" $6 \cdot 1$ tests per patient had been performed; when the final diagnosis was recorded as "probable" an average of $5 \cdot 1$ tests per patient had been performed; whereas when the final conclusion was that "no satisfactory diagnosis" had been reached the average number of tests was 6.0 per patient.

\section{Contribution of the Physical Examination and the Laboratory Investigations to the Diagnosis in Patients whose Histories were Unhelpful}

It seems reasonable to include in the group of patients with "unhelpful" histories those in whom the physicians recorded "don't know" with a confidence score of 8,9 , or 10 under "diagnosis after taking the history." There were eight such patients, and in two of these the physical examination allowed a diagnosis to be made (aortic stenosis in one patient, and osteoarthritis of the knee with phlebitis in the other). In the other six patients, however, the physicians still recorded "don't know" with a confidence score of 8,9 , or 10 under "diagnosis after physical examination." Laboratory investigations showed one of these six patients to have myeloma, but in the other five no definite organic cause for their complaint had been identified at the final review.

\section{Management of the Patient}

Table III shows how the patients were being managed two months after their visit to the outpatient clinic. In 60 patients their mode of management had been correctly predicted as soon as the history had been taken. In seven patients the physical examination had caused the physician to change his prediction (in five patients the examination had made him think that the disease was less serious, and in two that it was more serious) and in one of these patients the subsequent laboratory investigations then led to a further change in the method of management.

In 14 patients the result of the investigations led to a modification of the physicians' predicted management. In six of these it had initially been predicted that the patient would need to be 
followed up in the outpatient clinic without treatment, and as a result of the investigations the patients were able to be returned to the care of their general practitioners. In five patients there had been initial predictions of outpatient follow-up, but the patients subsequently needed to be referred to other clinics. In three patients the initial prediction of the need for outpatient treatment was altered in that two patients were returned to their general practitioners while one was admitted to hospital.

TABLE III-Management of Patients Two Months after their First Clinic Visit

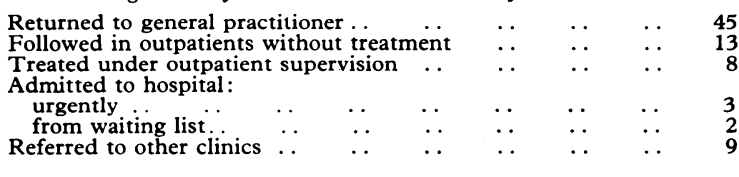

\section{Discussion}

Whether there is such a thing as a "typical" general medical outpatient clinic is a matter for debate, but the 80 patients reported in this study include a wide cross-section of outpatient medical practice. In 66 of these 80 patients the medical history provided enough information to make an initial diagnosis of a specific disease entity which agreed with the one finally accepted. The physical examination was useful in making the diagnosis in only seven patients, though in 25 patients it served to change the physician's confidence in the diagnosis which he had already reached on the basis of the history. The patients in whom the physical examination was most helpful all had diseases of the cardiovascular system. Surprisingly, the predictions about future management made after taking the history were less accurate than was the diagnosis made at this stage, the correct prediction being made in only 60 of the 80 patients. The physical examination led to a correct prediction in a further 12 patients.

No attempt has been made to estimate the cost of the different stages of the making of a diagnosis, but the laboratory investigations clearly have a low cost-effectiveness. Though $24 \%$ of the tests ordered gave abnormal results, in only seven patients did the investigations change the diagnosis which had been reached after history taking and physical examination. While the investigations changed the predicted management in 14 patients, only in eight did they lead to further action on the part of the hospital.

It is clear that physicians take widely different attitudes towards investigations, some relying on them much more heavily than others. In view of the increasing cost and complexity of investigations this area evidently requires more detailed investigation, and the reason why physicians order investigations will have to be more clearly defined if we are to have any form of audit.

Some physicians, for example, might consider a chest $x$-ray an essential investigation in all patients as part of a screening programme, while others might argue that haemoglobin must always be measured because anaemia, unlike lung cancer, is an essentially treatable condition, which must not be missed. Another purpose of investigation is to avoid the observer error inherent in all clinical observations. It is possible that some physicians order tests so that a definite statement of some aspect of a patient's condition at that time is available in the records. Further studies of physicians' attitudes to the use of investigations are clearly necessary.

Our study was limited to new patients presenting in a medical outpatient clinic, and our findings must not be extrapolated to other situations. We have demonstrated the value of the history only in the particular circumstance of a patient referred by a general practitioner because of one or more specific complaints which were of a relatively non-urgent nature; obviously, physical signs may be of paramount importance in making a diagnosis in emergencies. If, however, in a medical outpatient clinic the diagnosis can be made and the management of the patient can be accurately forecast on the basis of the history in three-quarters of the new patients seen, then there are some important implications.

Firstly, physicians can allocate the relative time spent taking the history and examining the patient with some confidence, knowing that extra time spent on the history is likely to be more profitable than extra time spent on the physical examination. Secondly, more emphasis must be placed on teaching students how to take accurate histories in a medical clinic, and proportionately less on showing them how to elicit physical signs. Thirdly, more emphasis must be placed on research into communication between the patient and his physician, and perhaps less emphasis is needed on the development of new laboratory services. Fourthly, there are implications for the planning of medical outpatient departments. There needs to be more emphasis on space for interviewing patients, and proportionately less on space for examining them.

Our findings also have implications for the number of follow-up appointments that need to be given to patients who seem to present diagnostic problems. It seems that if the physician is still in considerable doubt about the diagnosis after the history has been taken and the patient has been examined, then laboratory investigations are unlikely to be helpful.

We are grateful to Sir George Pickering and the physicians on his staff who co-operated with this study.

\section{Reference}

Platt, R., Manchester University Medical School Gazette, 1947, 27, 139. 